\title{
Driving-induced crossover: from classical criticality to self-organized criticality
}

\author{
Francisco-José Pérez-Reche, ${ }^{1,2}$ Lev Truskinovsky, ${ }^{2}$ and Giovanni Zanzotto ${ }^{3}$ \\ ${ }^{1}$ Department of Chemistry, University of Cambridge, Cambridge, CB2 1EW, UK \\ ${ }^{2}$ Laboratoire de Mécanique des Solides, CNRS UMR-7649, \\ Ecole Polytechnique, Route de Saclay, 91128 Palaiseau, France \\ ${ }^{3}$ Dipartimento di Metodi e Modelli Matematici per le Scienze Applicate, \\ Università di Padova, Via Trieste 63, 35121 Padova, Italy
}

\begin{abstract}
We propose a spin model with quenched disorder which exhibits in slow driving two drastically different types of critical nonequilibrium steady states. One of them corresponds to classical criticality requiring fine-tuning of the disorder. The other is a self-organized criticality which is insensitive to disorder. The crossover between the two types of criticality is determined by the mode of driving. As one moves from "soft" to "hard" driving the universality class of the critical point changes from a classical order-disorder to a quenched Edwards-Wilkinson universality class. The model is viewed as prototypical for a broad class of physical phenomena ranging from magnetism to earthquakes.
\end{abstract}

PACS numbers: 05.70.Jk,64.60.My,64.70.K,64.60.av

The study of criticality in externally driven inhomogeneous systems has attracted much attention in the last two decades [1]. Such systems exhibit rate independent dissipation and are widely used to model hysteretic phenomena and intermittency associated with magnetism, superconductivity, porous flow, fracture, friction, plasticity and martensitic phase transitions 2]. It has been realized that in most cases scaling emerges as an interplay between quenched disorder, extremal dynamics, and quasi-static driving [24]. Within this general framework the theoretical work has been mostly focussed on two types of models. In models of the Random Field Ising (RFIM) type, the critical behavior requires fine tuning of the amount of disorder $r$ and the intermittent events (avalanches) are scale-free only at a certain $r=r_{o}$ [1]. An alternative approach links criticality to a pinningdepinning (PD) transition where the disorder $r$ is an irrelevant parameter [3]. It has been established that criticality in the first class of models is classical, as in second order phase transitions [1], while in the second class it is self tuning in the sense that infinitely slow driving brings the system automatically on a critical manifold [4, 5, 6].

The two approaches are fundamentally different. The first model describes regimes with dominating nucleation, while the second one deals exclusively with propagation. It is then not surprising that the resulting criticality is different. In the RFIM the emerging scaling has been explained by the existence of a classical critical point of the order-disorder (OD) type. On the contrary, in $\mathrm{PD}$ theory one encounters a range of universality classes none of which can be formally reduced to OD. A relation between the two types of critical phenomena has been, however, anticipated. Previous work has shown that the presence of a nonlocal demagnetizing field of antiferromagnetic nature (as in soft magnets) can self tune the RFIM to display front-propagation critical exponents [7, 8]. In the PD framework similar 'self-tuning', often interpreted as self organized criticality (SOC), is obtained if the system is driven through a 'weak spring' which provides an explicit feedback mechanism [9]. In this letter we use these insights to develop the first unifying model which displays a crossover between the OD and quenched Edwards-Wilkinson (QEW) universality classes. We show that such crossover can be achieved experimentally by modifying the properties of the external driving. Since the QEW model is equivalent to the Oslo rice pile model [12] and is therefore paradigmatic for SOC, we are essentially dealing here with a fundamental relation between OD and SOC.

We base our model on the observation that solids can be deformed either by applying a force (soft device), or by controlling surface displacements (hard device) [13, 14]. Both driving mechanisms, soft and hard, can be handled simultaneously if the system has a finite 'elasticity'. To introduce this effect in a spin setting, consider a prototypical model which we call RSSM (Random Soft-Spin [15] or Snap-Spring [16, 17] Model). The main difference between the RSSM and its predecessor RFIM is the finite curvature of the energy wells and the presence of elastic barriers. While the role of the softness of the spins is known to be secondary in equilibrium and in the case of soft driving, where it can be accounted for through an appropriate 'dressing' of the underlying hard-spin model [15], the curvature of the wells becomes crucial in the case with hard driving where it plays the role of a regularization of the otherwise degenerate problem 14. Consider a set of $N$ bi-stable units located on the nodes $i=1,2, \ldots N$ of a cubic lattice with linear size $L=N^{1 / 3}$. The state of each snap-spring is characterized by a continuous scalar order parameter $e_{i}$ measuring the local 'strain'. The energy of the system is

$$
\phi=\frac{1}{N} \sum_{i=1}^{N} f_{i}\left(e_{i}\right)+\frac{1}{2 N} \sum_{i, j=1}^{N} K_{i j} e_{i} e_{j},
$$

where $f_{i}\left(e_{i}\right)$ is a double-well potential and $\mathbf{K}=\left\{K_{i j}\right\}$ is the interaction kernel with sufficient rate of decay to 
ensure convergence in the thermodynamic limit. In each well (one defined for $e_{i}<0$ and the other for $e_{i}>0$ ) we use the approximation $f_{i}\left(e_{i}\right)=\frac{1}{2}\left(e_{i}-s_{i}\right)^{2}-h_{i}^{\prime} e_{i}$, where $s_{i}= \pm 1$ is a spin variable and $\left\{h_{i}^{\prime}\right\}$ are random numbers representing quenched disorder. The system is loaded through an 'elastic' device with the energy $\phi_{d}=\frac{c}{2}(e-\bar{e})^{2}$, where $\bar{e}=(1 / N) \sum_{i} e_{i}$ is the average strain of the system of snap-springs, $c$ is the stiffness of the loading device and $e$ is the control parameter (global 'strain'). One obtains a hard device in the limit $c \rightarrow \infty$, and a soft device in the limit $c \rightarrow 0, e \rightarrow \infty$ with the stress $\sigma=c e$ fixed; the system will be driven quasi-statically by changing the control parameter $e$, if $c$ is finite, and $\sigma$, if $c=0$. We neglect thermal fluctuations $(T=0)$ and assume that the harmonic variables $e_{i}$ relax 'instantaneously' and can be adiabatically eliminated. By minimizing the total energy $\phi+\phi_{d}$ with respect to $e_{i}$ we obtain $e_{i}=\tilde{e}+\sum_{j=1}^{N}\left(J_{i j}-k / N\right) s_{j}+h_{i}$ where $\mathbf{J}=(\mathbf{1}+\mathbf{K})^{-1}$ is the effective interaction between the spin variables $s_{i}$. We impose periodic boundary conditions, meaning that $k_{\infty}=\sum_{j} J_{i j}$ does not depend on $i$, and use the notations $\tilde{e}=e\left[c k_{\infty}\left(c k_{\infty}+1\right)^{-1}\right], k=k_{\infty}\left[c k_{\infty}\left(1+c k_{\infty}\right)^{-1}\right]$, and $h_{i}=\sum_{j} J_{i j} h_{j}^{\prime}$.

Because of the absence of thermal fluctuations and to the separation of time scales between overdamped relaxation and driving, the system remains on a metastable branch $\left\{e_{i}\left(\left\{s_{k}\right\} ; e\right)\right\}$ corresponding to a particular local minimum of the total energy until the latter ceases to be stable [17]. When the instability condition $s_{i} e_{i}<0$ is reached for some $i$ the system jumps (through an avalanche) to another locally stable branch characterized by a different spin configuration $\left\{s_{i}^{\prime}\right\}$. We increase $e$ by driving the system from an initial stable configuration with $\left\{s_{i}=-1\right\}$ and assume that avalanches propagate (at constant $e$ ) with synchronous dynamics. For simplicity, we consider only nearest-neighbor interactions with $J_{i i}=J_{0}, J_{i j}=J_{1}$, and we set $J_{0}=J_{1}=1$ so that $k_{\infty}=7$. The renormalized disorder variables $\left\{h_{i}\right\}$ are drawn from a Gaussian distribution with zero mean and standard deviation $r$. Under these assumptions, the RSSM becomes formally equivalent to a nonlocal augmentation of the classical RFIM with demagnetizing factor $k[1,6,6,8,8,18]$.

At $k=0$ we expectedly observe an OD transition at $r_{o} \simeq 2.2$ which separates a 'popping' (POP) regime $\left(r>r_{o}\right)$ where all the avalanches are small from a 'snapping' (SNAP) regime $\left(r<r_{o}\right)$ where an infinite avalanche sweeps most of the system [1, 19]. The stress-strain curve $\sigma(e)=k_{\infty}^{-1}\left(\tilde{e}-k N^{-1} \sum_{i} s_{i}\right)$ is continuous in the POP regime and displays a macroscopic discontinuity of the strain in the SNAP regime [see Fig. 1. The strain discontinuity associated with the nucleation of the infinite avalanche occurs at a 'nucleation' stress $\sigma_{n}$ which decreases with $r$ as indicated in Fig. 2,

For non-zero $k$, the POP regime remains essentially unaltered. In contrast, stiffness has a non-trivial effect

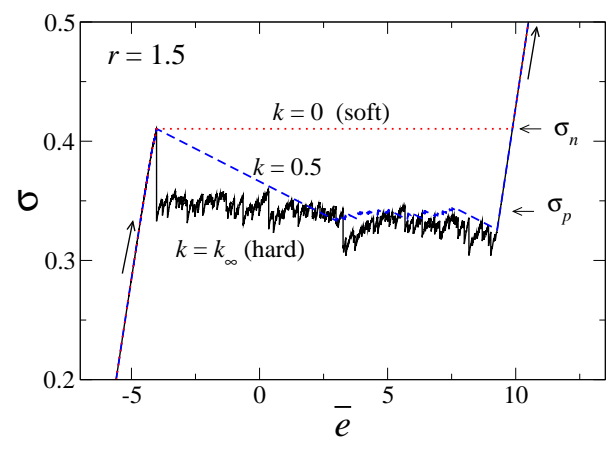

FIG. 1: Stress-strain curves for $k=0$ (dotted line), $k=$ 0.5 (dashed line), and $k=k_{\infty}$ (continuous line) for $r=1.5$ in a system with $L=64 ; \sigma_{p}$ and $\sigma_{n}$ are propagation and nucleation thresholds, respectively.

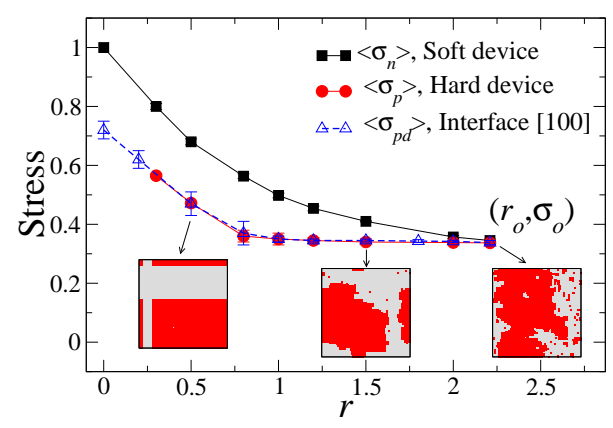

FIG. 2: Dependence on disorder of the averaged nucleation and propagation thresholds: $\left\langle\sigma_{n}\right\rangle$ (squares), $\left\langle\sigma_{p}\right\rangle$ at $k=k_{\infty}$ (circles), and $\left\langle\sigma_{p d}\right\rangle$ for an interface [100] (triangles). Insets: cross sections of the $3 \mathrm{D}$ system showing typical transformation domains for $k=k_{\infty}$. Darker and lighter colors indicate transformed regions $(s=+1)$ and untransformed regions $(s=-1)$.

over the SNAP regime observed at low disorders. In this case, a compact domain reminiscent of the infinite avalanche (as in the $k=0$ case) nucleates at the stress $\sigma_{n}$. The stress relaxes during the avalanche growth to satisfy the global driving constraint. When $k$ is below a certain threshold $k_{p}(r)$, the SNAP behavior remains qualitatively as in the $k=0$ case because the nonlocal constraint is still soft. In contrast, when $k>k_{p}(r)$, the first stress drop prevents the spanning avalanche from growing [Fig. 1]. The transformation induced by the subsequent increase of $e$ proceeds through the intermittent growth of the previously nucleated domain with untransformed system acting as a disordered background. The propagation is accompanied by stress oscillations around the propagation threshold $\sigma_{p}$ which remains stable during the whole yielding process. The average of $\sigma_{p}$ over disorder coincides with the depinning stress $\sigma_{p d}$ for a flat [100] interface artificially introduced and driven as in Refs. 20, 21. This is a clear evidence that systems with $k>k_{p}(r)$ reach a front-propagation regime which self-tunes exactly around the PD point. The surface mor- 

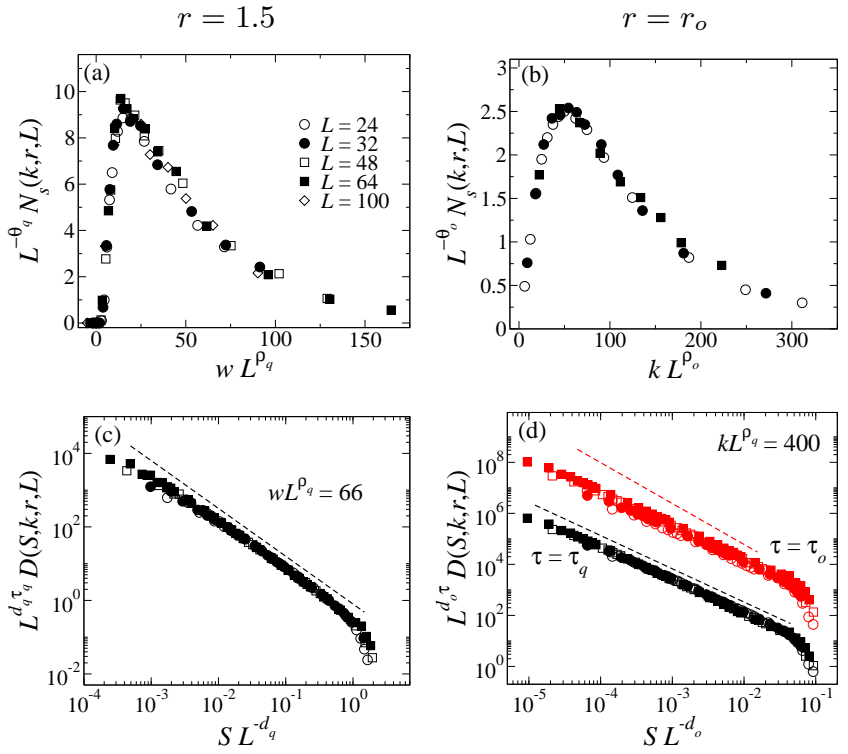

FIG. 3: Scaling collapse of $N_{s}(k, r, L)(\mathrm{a}, \mathrm{b})$ and $D(S ; k, r, L)$ (c,d) according to the hypotheses in Table I Different symbols correspond to different system sizes as indicated in the legend. Data in plots to the left (right) correspond to $r=1.5$ $\left(r=r_{o}\right)$. Data in (c) correspond to $w L^{\rho_{s}}=66$; in (d) to $k L^{\rho_{o}}=400$. The lower scaling collapse in $(\mathrm{d})$ is obtained with exponent $\tau_{q}$ while the upper one corresponds to $\tau_{o}$; dashed lines indicate the power-laws expected in the thermodynamic limit.

phology of the growing domain is faceted at low disorders $(r \lesssim 0.8$ for systems with $L=100)$ and is self-affine at intermediate disorders. According to previous studies [21, 22], the faceted morphology is unstable giving rise to self-affine morphology in the thermodynamic limit for any finite $r$. Given the self-affine character of the boundary of the nucleated domain in the case of intermediate disorders and the short-range character of the involved interactions, one expects to observe critical scaling of the QEW (quenched Edwards-Wilkinson) universality class [3].

We check the validity of this assumption by analyzing the scaling properties of the number of spanning avalanches $N_{s}(k, r, L)$ and the distribution $D(S ; k, r, L)$ of avalanche sizes $S$. Following the arguments given in Refs. [19], we consider contributions to $N_{s}$ of avalanches spanning along 1 or 2 dimensions only. Table I summarizes the action of the Renormalization Group ( $R G$ ) transformation on the parameters of the system and the resulting scaling hypotheses. The scaling variables measuring the distance to the OD critical point are $u=r-r_{o}$ and $k$. The distance to the QEW critical manifold is $w=k-k_{p}(r)$.

Scaling collapse for $N_{s}(k, r, L)$ close to the line $k_{p}(r)$, shown in Fig. 3(a), confirms the validity of our scaling hypothesis. We have checked that QEW scaling persists over a finite interval of disorders $r<r_{o}$. QEW scal- (a)

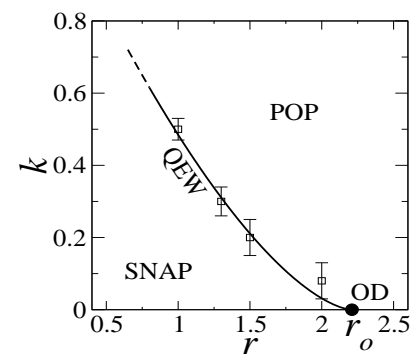

(b)

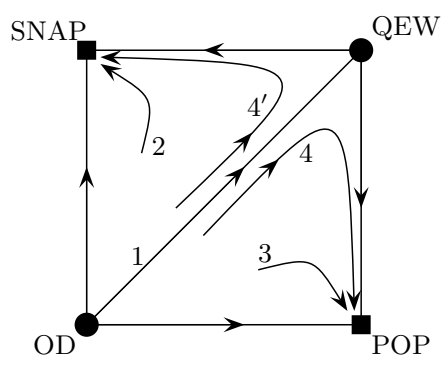

FIG. 4: (a) Schematic phase diagram for the RSSM (thermodynamic limit). Criticality of the OD type is associated with the point $r=r_{o}, k=0$. Criticality of the QEW type is expected on the line $k_{p}(r)$ separating SNAP and POP regimes; symbols display estimations of $k_{p}(r)$ from scaling collapses. (b) Schematic RG flow for the RSSM model. Separatrix 1 going from the neighborhood of the OD fixed point towards the QEW fixed point indicates the QEW critical manifold. The RG-flow towards SNAP and POP regimes is indicated by arrows 2 and 3, respectively. Lines 4 and $4^{\prime}$ correspond to systems which display SOC as an intermediate asymptotics (QEW exponents with supercritical or subcritical cutoffs for $k<k_{p}(r)$ and $k>k_{p}(r)$, respectively).

ing fails for disorders close to $r_{o}$ (above $r \sim 2$ for our system sizes) due to crossover to the OD critical regime. Fig. 3.(b) displays the scaling collapse at $u=0$ which generates the exponents $\rho_{o}$ and $\theta_{o}$ given in Table 1 . The value of $\theta_{o}$ which, in contrast to $\theta_{q}$ is positive, agrees with previous estimates for the RFIM 19]. Fig. 3(c) presents the scaling collapse of $D(S ; k, r, L)$ for $r=1.5$ corresponding to a particular section of the function $\tilde{D}\left(S L^{-d_{q}}, w L^{\rho_{q}}\right)$ at constant $w L^{\rho_{q}}$. The resulting exponents $d_{q}$ and $\tau_{q}$ listed in Table \ do not depend on the selected section and are in agreement with previous estimates from frontpropagation models [4, 7, 21]. This confirms once again that the propagation regime is of the QEW class; the analysis of statistics of durations, omitted here, also supports this interpretation. The value of $d_{q}$, giving the dimension of the avalanches in the propagation regime, is consistent with $d-1$, which confirms the self-affine morphology of the propagating domain boundary [21]. The distribution $D(S ; k, r, L)$ at $r=r_{0}$ scales with exponents previously reported for the OD universality class when $k$ is very small. The exponent $\tau_{o}$ displays a considerable crossover to QEW when the stiffness becomes relatively large. For instance, Fig. 3(d) shows that the scaling of $D(S ; k, r, L)$ at $k L^{\rho_{o}}=400$ is better with exponent $\tau_{q}$ than with $\tau_{o}$.

In Fig. 4(a) we present the phase diagram in the $(r, k)$ plane showing stable SNAP and POP regimes separated by the QEW line $w=0$. This line describes QEW behavior and it ends in a point corresponding to the OD regime. The variety of observed non-equilibrium steady states can be explained if one assumes the existence of four fixed points for the RG flow, schematically depicted 
TABLE I: Scaling of the relevant quantities under a RG transformation with blocking parameter $b$ and values of the associated critical exponents (close to OD (subindex ' $o$ ') and to QEW (subindex ' $q$ ')).

\begin{tabular}{|c|c|c|c|c|}
\hline & \multicolumn{2}{|r|}{ OD } & \multicolumn{2}{|c|}{ QEW } \\
\hline & $\mathrm{RG}$ & exponents & $\mathrm{RG}$ & exponents \\
\hline System size, $L$ & $L(b)=b^{-1} L$ & & $L(b)=b^{-1} L$ & \\
\hline Stiffness, $k$ & $k(b)=b^{\rho_{o}} k$ & $\rho_{o}=1.3 \pm 0.3$ & $w(b)=b^{\rho_{q}} w$ & $\rho_{q}=0.8 \pm 0.2$ \\
\hline Disorder, $r$ & $u(b)=b^{1 / \nu_{o}} u$ & $\nu_{o}=1.2 \pm 0.1([19])$ & Irrelevant & \\
\hline Size, $S$ & $S(b)=b^{-d_{o}} S$ & $d_{o}=2.78 \pm 0.05([19])$ & $S(b)=b^{-d_{q}} S$ & $d_{q}=2.0 \pm 0.1$ \\
\hline$N_{s}(k, r, L)$ & $N_{s}(b)=b^{-\theta_{o}} N_{s}$ & $\theta_{o}=0.10 \pm 0.02([19])$ & $N_{s}(b)=b^{-\theta_{q}} N_{s}$ & $\theta_{q}=-0.2 \pm 0.06$ \\
\hline$D(S ; k, r, L)$ & $D(b)=b^{\tau_{o} d_{o}} D$ & $\tau_{o}=1.6 \pm 0.06([1])$ & $D(b)=b^{\tau_{q} d_{q}} D$ & $\tau_{q}=1.3 \pm 0.06$ \\
\hline$N_{s}(k, r, L)$ & \multicolumn{2}{|c|}{$L^{\theta_{o}} \hat{N}_{s}\left(k L^{\rho_{o}}, k u^{-\rho_{o} \nu_{o}}\right)$} & \multicolumn{2}{|c|}{$L^{\theta_{q}} \tilde{N}_{s}\left(w L^{\rho_{q}}\right)$} \\
\hline$D(S ; k, r, L)$ & \multicolumn{2}{|c|}{$L^{-d_{o} \tau_{o}} \hat{D}\left(S L^{-d_{o}}, k L^{\rho_{o}}, k u^{-\rho_{o} \nu_{o}}\right)$} & \multicolumn{2}{|c|}{$L^{-d_{q} \tau_{q}} \tilde{D}\left(S L^{-d_{q}}, w L^{\rho_{q}}\right)$} \\
\hline
\end{tabular}

in Fig. 4(b). The OD regime is associated with a fully repulsive critical point which can be reached only by tuning all four parameters: $\sigma=\sigma_{c}, r=r_{o}, k=0$, and $L^{-1}=0$. In contrast, QEW is a saddle point with a stable manifold which governs the large scale behavior of the systems with $r<r_{o}, \sigma=\sigma_{p d}(r), k=k_{p}(r)$, and $L^{-1}=0$. As we have seen the condition $\sigma=\sigma_{p d}(r)$ is reached automatically during the self-organized propagation regime with $k \geq k_{p}(r)$; the corresponding systems lay on the critical manifold connecting OD and QEW points. The large scale behavior for systems located away from the critical manifold is governed either by SNAP (arrow 2) or by POP (arrow 3) fixed points which are trivial attractors in the RG sense. While the self-organized propagation regime is strictly critical only for $k=k_{p}(r)$, our numerical simulations show that the system exhibits truncated power law scaling with QEW exponents in a broad range of parameters around the line $k_{p}(r)$ (arrows 4 and $4^{\prime}$ in Fig. 4(b)). This observation suggests that SOC can be viewed as an intermediate asymptotics. In general, since in real systems stiffness is finite and disorder is generic, we anticipate the power law structure of critical fluctuations to be in most cases of the PD rather than of the OD type.

We acknowledge helpful discussions with M.L. Rosinberg, S. Roux, and G. Tarjus and partial funding from EU contract MRTN-CT-2004-505226.

* Electronic address: fjp23@cam.ac.uk

[1] J. P. Sethna, K. A. Dahmen, and C. R. Myers, Nature (London) 410, 242 (2001); J. P. Sethna, K. A. Dahmen, and O. Perković, in The science of hysteresis II, edited by G. Bertotti and I. D. Mayergoyz (Academic Press, Amsterdam, 2006).

[2] M. Kardar, Phys. Rep. 301, 85 (1998); D. S. Fisher, Phys. Rep. 301, 113 (1998); M. Zaiser, Adv. Phys. 55, 185 (2006); M. J. Alava, P. Nukala, and S. Zapperi, Adv. Phys. 55, 349 (2006).

[3] O. Narayan and D. S. Fisher, Phys. Rev. B 48, 7030 (1993); P. Chauve, P. Le Doussal, and K. J. Wiesse, Phys. Rev. Lett. 86, 1785 (2001).

[4] G. Durin and S. Zapperi, in The science of hysteresis,
II, edited by G. Bertotti and I. D. Mayergoyz (Academic Press, Amsterdam, 2006).

[5] K. A. Dahmen and Y. Ben-Zion, in Encyclopedia of Complexity and Systems Science (Springer, 2008).

[6] J. S. Urbach, R. C. Madison, and J. T. Market, Phys. Rev. Lett. 75, 276 (1995).

[7] M. C. Kuntz and J. P. Sethna, Phys. Rev. B 62, 11699 (2000).

[8] J. H. Carpenter et al., Phys. Rev. B 72, 052410 (2005).

[9] B. Alessandro et al., J. Appl. Phys. 68, 2901 (1990).

[10] P. Cizeau et al., Phys. Rev. Lett. 79, 4669 (1997).

[11] R. Dickman et al., Braz. J. Phys. 30, 27 (2000).

[12] M. Paczuski and S. Boettcher, Phys. Rev. Lett. 77, 111 (1996); G. Pruessner, Phys. Rev. E 67, 030301R (2003).

[13] G. Puglisi and L. Truskinovsky, J. Mech. Phys. Solids 48, 1 (2000); E. Bonnot et al., Phys. Rev. B 76, 064105 (2007).

[14] G. Bertotti, Hysteresis in magnetism (Academic Press, San Diego, 1998); X. Illa, M.L. Rosinberg, and E. Vives, Phys. Rev. B 74, 224403 (2006); X. Illa et al., Phys. Rev. B 74, 224404 (2006).

[15] D. J. Bergman and B. I. Halperin, Phys. Rev. B 13, 2145 (1976); K. A. Dahmen and J. P. Sethna, Phys. Rev. B 53, 14872 (1996).

[16] I. Müller and P. Villaggio, Arch. Rat. Mech. Anal. 65, 25 (1977); B. Fedelich and G. Zanzotto, J. Nonlinear Sci. 2, 319 (1992).

[17] L. Truskinovsky and A. Vainchtein, J. Mech. Phys. Solids 52, 1421 (2004); G. Puglisi and L. Truskinovsky, J. Mech. Phys. Solids 53, 655 (2005).

[18] S. Queiroz, Phys. Rev. E 77, 021131 (2008).

[19] F. J. Pérez-Reche and E. Vives, Phys. Rev. B 67, 134421 (2003), ibid. 70, 214422 (2004).

[20] L. Roters et al., Phys. Rev. E 60, 5202 (1999).

[21] H. Ji and M. O. Robbins, Phys. Rev. B 46, 14519 (1992); B. Koiller and M. O. Robbins, Phys. Rev. B 62, 5771 (2000).

[22] J. Bouchaud and A. Georges, Phys. Rev. Lett. 68, 3908 (1992); T. Emig and T. Nattermann, Eur. Phys. J. B 8, 525 (1999).

[23] P. Bak, C. Tang, and K. Wiesenfeld, Phys. Rev. Lett. 59, 381 (1987); K. Chen, P. Bak, and S. Obukhov, Phys. Rev. A 43, 625 (1991); F. J. Pérez-Reche, L. Truskinovsky, and G. Zanzotto, Phys. Rev. Lett. 99, 075501 (2007).

[24] We exclude here similar critical phenomena which do not require quenched disorder, e.g. [23]. 